\title{
Canonical Correlations Between Body Posture Variables and Postural Stability in Children with Scoliosis and Scoliotic Posture
}

\author{
Jacek Wilczyński ${ }^{1}$, Natalia Habik², Katarzyna Bieniek², Sylwia Janecka², Przemysław Karolak ${ }^{2} \&$ Igor $^{2}$ \\ Wilczyński ${ }^{4}$ \\ ${ }^{1}$ Head of Posturology Department, Hearing and Balance Rehabilitation, Faculty of Medicine and Health \\ Sciences, Jan Kochanowski University in Kielce, Poland \\ ${ }^{2}$ Faculty of Medicine and Health Sciences, Jan Kochanowski University in Kielce, Poland \\ ${ }^{3}$ Faculty of Rehabilitation, University School of Physical Education in Krakow, Kraków, Poland \\ ${ }^{4}$ Outpatient Rehabilitation Centre for Children, State Health Care Center in Starachowice, Poland \\ Correspondence: Jacek Wilczyński, Head of Posturology Department, Hearing and Balance Rehabilitation, \\ Faculty of Medicine and Health Sciences, Jan Kochanowski University, Kielce, Al. IX Wieków Kielc 19, 25-317 \\ Kielce, Poland. Tel: 48-603-703-926. E-mail: jwilczyński@onet.pl
}

Received: April 13, 2018

doi:10.5539/mas.v12n6p58
Accepted: April 25, $2018 \quad$ Online Published: May 21, 2018

URL: https://doi.org/10.5539/mas.v12n6p58

\begin{abstract}
Background: The aim of the study was to analyse the correlation between body posture variables and postural stability in children with scoliosis and scoliotic posture.

Methods: Spinal examination photogrammetry used the photometric Moiré method. Based on the angle size of the of spinal curvature, scoliotic posture was determined: $1-9^{\circ}$, and scoliosis: $\geq 10^{\circ}$. Postural reactions were tested using the Tecnobody ST 310 Plus Stability System platform. Children attended therapy at the Inter-school Centre of Corrective and Compensatory Gymnastics in Starachowice The study was conducted in June 2011. There were 21 children with scoliotic posture (7\%) and 7 with scoliosis (25\%).

Results: In the canonical analysis of body posture variables, the highest share comprised of: trunk inclination angle, alpha angle, chest kyphosis angle, length of lumbar lordosis, length of lumbar lordosis/total spine length, shoulder asymmetry - right higher, shoulder asymmetry - left higher, absolute of pelvis tilt angle, coefficient of shoulder asymmetry relative to $\mathrm{C}_{7}$, primary curvature angle, length of secondary curvature/total spine length, depth of secondary curvature/total spine length.
\end{abstract}

Significance: High values of canonical correlation coefficients, despite lack of significance, indicate the possibility of strong a correlation between body postural variables and postural stability that can be demonstrated with a greater sample size.

Keywords: scoliotic posture, idiopathic scoliosis, postural stability, posturography computer

\section{Introduction}

Children with scoliosis compensate for incorrect alignment of individual body parts which allows them to maintain balance [1,2]. Postural stability plays an important here [3,4]. Stabilization is considered in the biomechanical aspect, but also in part of CNS movement control [5,6]. For a long time, it was thought that the superficial muscles of the trunk were responsible for stabilization of the body [7,8]. A modern approach to the problem of active stabilization of the spine originates in Bergmark's ideas [9]. Stabilization of the body is associated with postural reactions which are its resultant and are part of balance [10,11]. An overall assessment of postural stability is provided via posturographic testing $[12,13]$. This is possible through the analysis of reactions and postural strategies that form the basis of systems maintaining the body balance [14]. Equilibrium is a specific state of the postural system that characterizes the vertical orientation of the body achieved by aligning the forces acting on the body and their moments [15]. Balance provides the nervous system with reflex antigravity muscles [16]. There are ways to build or correct this stabilization by normalizing postural tension and thus, scoliosis re-education [17]. The aim of the study was to analyse the correlation between body posture variables and postural stability in children with scoliosis and scoliotic posture. 


\section{Methods}

\subsection{The Population}

The study included 28 girls, aged 7-18 with scoliotic posture and idiopathic scoliosis. The selection of test subjects was not random. Children attended therapy at the Inter-school Centre of Corrective and Compensatory Gymnastics school. The study was conducted in June 2011.

\subsection{Information Collection}

All procedures performed in test involving human participants were in accordance with the ethical standards of the institutional and/or national research committee and with the 1964 Helsinki declaration and its later amendments or comparable ethical standards. The guardians of the children were informed about the purpose of the study and expressed written consent for their children's participation in the study. The study was non-invasive and free of charge. The patients willingly participated in the study, and perceived it as a concern about their state of health.

\subsection{Measurement Scales}

The photogrammetric Moiré method was used in postural tests. Spinous processes from C7 to S1 were marked on the back of tested subject using a marker, as well as the acromion, lower angles of the shoulder blades and the posterior superior iliac spine. The subject assumed a habitual position at the rear of the device at a distance of 3.2 $\mathrm{m}$. Stripes were projected onto the back, and the focusing of the lens allowed the Moiré image to be obtained. The image of the spine was received by an optical system with a camera, then it was passed onto to the analogue monitor and finally, to the computer. In this way, a three-dimensional image of the back was formed. The following parameters of the posture in the sagittal plane were analysed: total spine length $(\mathrm{MM})$, trunk inclination angle $\left(^{\circ}\right)$, absolute trunk inclination angle $\left(^{\circ}\right)$, alpha angle $\left({ }^{\circ}\right)$, beta angle $\left({ }^{\circ}\right)$, gamma angle $\left({ }^{\circ}\right)$, length of chest kyphosis $(\mathrm{MM})$, length of chest kyphosis/total spine length (\%), angle of chest kyphosis $\left({ }^{\circ}\right)$, actual angle of chest kyphosis $\left({ }^{\circ}\right)$, actual angle of chest kyphosis/total spine length (\%), depth of chest kyphosis (mm), depth of chest kyphosis/total spine length (\%), absolute value of chest kyphosis depth/total spine length (\%), length of lumbar lordosis $(\mathrm{mm})$, length of lumbar lordosis/total spine length $(\%)$, lumbar lordosis angle $\left({ }^{\circ}\right)$, actual angle of lumbar lordosis $\left({ }^{\circ}\right)$, actual angle of lumbar lordosis/total spine length (\%), depth of lumbar lordosis (mm), depth of lumbar lordosis/total spine length (\%). Next, primary and secondary spinal curvature parameters were analysed: shoulder asymmetry - right higher $(\mathrm{mm})$, shoulder asymmetry - left higher $(\mathrm{mm})$, shoulder line angle $\left({ }^{\circ}\right)$, shoulder blade asymmetry - right higher $(\mathrm{mm})$, shoulder blade asymmetry - left higher $(\mathrm{mm})$, pelvis tilt angle $\left(^{\circ}\right)$, absolute value of pelvis tilt angle $\left({ }^{\circ}\right)$, pelvis rotation angle $\left({ }^{\circ}\right)$, pelvis rotation $\left({ }^{\circ}\right)$, shoulder/pelvis asymmetry coefficient $(\%)$, shoulder asymmetry coefficient $-\mathrm{KK}$ point $(\%)$, shoulder $/ \mathrm{C}_{7}$ asymmetry coefficient $(\%)$, length of curvature $(\mathrm{mm})$, length of curvature/total spine length (\%), depth of curvature $(\mathrm{mm})$, depth of curvature/total spine length (\%), curvature angle $\left({ }^{\circ}\right)$, absolute value of curvature angle $\left({ }^{\circ}\right)$. Based on the size of the spinal curvature, the following were distinguished: scoliotic postures: $1-9^{\circ}$ and scoliosis: $\geq 10^{\circ}$. Postural stability was tested using the staticdynamic ST 310 Plus Stability System platform made by Tecnobody. Testing on the platform consisted of continuous monitoring of the center-of-foot pressure (COP). By recording the postural sways as a function of time, accurate information about the postural system was obtained. The standard free-standing stability test (the Romberg test) was performed. The trial consisted of two successive 30-second tests: the first with open eyes (OE - open eyes) and the second with closed eyes (CE - closed eyes). The studied subject assumed a habitual position with arms lowered along the torso and the head straightened. The test begun when the subject assumed the position, and the center-of-pressure (COP) was displayed on the screen. The following parameters were recorded for the measurement of COP: average COP X in relation to the platform axis determines lateral $\mathrm{X}$ coordinates $(\mathrm{mm})$; average COP Y in relation to the platform axis determines frontal-posterior Y coordinates $(\mathrm{mm})$; medium-lateral standard deviation $\mathrm{X}$ is the mean oscillation along the $\mathrm{X}$ axis $(\mathrm{mm})$ and the mean lateral deflection $(\mathrm{mm})$, i.e. the average distance between the extreme deflection from the COP in the lateral plane, forward-backward standard deviation $\mathrm{Y}$ is the mean oscillation along the $\mathrm{Y}$ axis $(\mathrm{mm})$ and the mean forward-backward deflection $(\mathrm{mm})$ - the average distance between the extreme deflection from the COP in the sagittal plane, average forward-backward speed Y, i.e. the mean speed of oscillation along the $\mathrm{Y}$ axis $(\mathrm{mm} / \mathrm{s})$. It is the quotient of the length of deflection from the COP during the test which indirectly informs about the dynamics of the postural stability regulation process in a standing position, average medium-lateral speed $X$, i.e. the mean speed of oscillation along the $X$ axis $(\mathrm{mm} / \mathrm{s})$. It is the quotient of the length of deflection from the COP during the test which indirectly informs about the dynamics of the postural stability regulation process in a standing position, perimeter. This is the total length of the COP path in both planes during oscillation $(\mathrm{mm})$, ellipse area. This is the total area the COP drew in both planes during oscillation $\left(\mathrm{mm}^{2}\right)$, perimeter ratio is the ration of the perimeter with open $(\mathrm{OE})$ and closed $(\mathrm{CE})$ eyes 
in the Romberg test, area ratio is the ratio of the ellipse area with eyes closed (OE) to the area with open eyes (OE) in the Romberg test. Individuals with postural disorders generally show higher values for all the listed parameters. Similarly, higher levels of sways occur in children. Variables were verified for normal distribution using the Shapiro-Wilk test. In order to distinguish variables without correlations, factor analysis was conducted. The correlation between scoliotic and postural stability variables was defined by canonical correlations. Significant levels were at $p<0.05$.

\section{Results}

There were 21 children with scoliotic posture (7\%) and 7 with scoliosis $(25 \%)$. In order to reduce the large number of random variables, canonical correlation analysis was preceded by factorial analysis. This analysis was also performed to determine the structure of the correlation between the tested variables. In this way, the observed variable $(\mathrm{R})$ factors $(\mathrm{F})$, which are linear combinations of these variables, are replaced.

\subsection{Descriptive Analysis}

The factorial loads for particular variables determine the extent to which the factor represents a given variable. It is assumed that the variables for which the absolute values of the factor loadings are greater than 0.7 , are significant for the factor. The factor for these variables is representative and the set of variables can be replaced by it. It was assumed that only variables associated with large values of factor loads carry the right amount of information, i.e. they have a large amount of variability, and these variables should be considered in further analysis of the correlation. Only factors that accounted for the largest variance were considered. The criterion was used to select those factors whose share in total variance was significantly higher than the others. The measure of the share of a factor in the total variance was its eigenvalue. Using the scree plot to determine their number was one of the methods for selecting factors. Factors of high volumetric variables were also taken into account in their selection. The factorial array was used in further analysis in place of the values of the variables directly observed. In this way, the determinants were used in multiple regression analysis, both as dependent and independent variables. The following five factors characterizing body posture in the sagittal plane were distinguished: Factor 1: angle of chest kyphosis ( $\mathrm{LC}=-0.882)$, Factor 2: trunk inclination angle ( $\mathrm{LC}=-0.944)$, Factor 3: alpha angle ( $\mathrm{LC}=0.919)$, Factor 4: length of lumbar lordosis ( $\mathrm{LC}=0.960)$, Factor 5: actual angle of lumbar lordosis/total spine length ( $\mathrm{LC}=-0.847)$. The share of these five factors in the total variance was significantly higher than the others. Selected orthogonal factors accounted for $89.3 \%$ of the total variance (Table 1). Then, from among the normalized scoliotic variables, the following seven factors were identified: Factor 1: length of secondary curvature/total spine length (LC $=0.973$ ), Factor 2: primary curvature angle $(\mathrm{LC}==-0.933)$, Factor 3: depth of primary curvature/total spine length ( $\mathrm{LC}=0.967)$, Factor 4: coefficient of shoulder asymmetry relative to $\mathrm{C}_{7}(\mathrm{LC}=0.867)$, Factor 5 : absolute value of pelvis tilt angle $\left({ }^{\circ}\right)(\mathrm{LC}=0.797)$, Factor 6: shoulder blade asymmetry - left higher $(\mathrm{mm})(\mathrm{LC}=0.786)$, Factor 7 : shoulder asymmetry - right higher ( $\mathrm{LC}=0.834)$. The emerged orthogonal factors accounted for $86.3 \%$ of the total variance (Table 2). Similarly, five factors of postural stability with open eyes were identified: Factor 1: mediumlateral standard Deviation X (OE) ( $\mathrm{LC}=0.961)$, Factor 2: absolute average of COP X (OE) ( $\mathrm{LC}=-0.933)$, Factor 3: forward-backward standard deviation $\mathrm{Y}(\mathrm{OE})(\mathrm{LC}=0.883)$, Factor 4: average forward-backward speed $\mathrm{Y}(\mathrm{OE})$ ( $\mathrm{LC}=0.817)$, Factor 5: average COP X (OE) ( $\mathrm{LC}=-0.829)$. The factors accounted for $92.6 \%$ of the total variance (Table III). Then, among the normalized variables of postural stability with closed eyes (CE), five factors were identified: Factor 1: Factor 1: medium-lateral standard deviation $\mathrm{X}(\mathrm{OE})(\mathrm{LC}=0.961)$, Factor 2: absolute value of average COP X (OE) (LC=-0.886), Factor 3: forward-backward standard deviation $\mathrm{Y}(\mathrm{OE})(\mathrm{LC}=0.883)$, Factor 4: average forward-backward speed $\mathrm{Y}(\mathrm{OE})(\mathrm{LC}=0.817)$, Factor 5: average COP X $(\mathrm{OE})(\mathrm{LC}=-0.829)$. The factors accounted for $92.6 \%$ of the total variance (Tab. 3). Then, among the normalized variables of postural stability with closed eyes (CE), five factors were identified: Factor 1: average forward-backward speed Y (CE) (LC=0.974), Factor 2: absolute value of average COP Y (CE) (LC=0.927), Factor 3: absolute value of average COP X (CE) $(\mathrm{LC}=-0.902)$, Factor 4: average COP X (CE) ( $\mathrm{LC}=0.937)$, Factor 5: medium-lateral standard deviation $\mathrm{X}(\mathrm{CE})$ $(\mathrm{LC}=0.515)$. The extracted orthogonal factors accounted for $89.9 \%$ of the total variance (Table III). In the canonical correlation related to body posture variables, the largest share comprised of: trunk inclination angle OE (0.555), CE (0.243), alpha angle OE (-0.070), CE (-0.422), chest kyphosis angle OE (0.216), CE (-0.347), length of lumbar lordosis OE (0.409), CE (0.107), length of lumbar lordosis/total spine length OE (-0.339), CE (0.864), shoulder asymmetry - right higher OE (-0.199), CE (-0.271), shoulder asymmetry - left higher OE (-0.641), CE (0.392), absolute of pelvis tilt angle $\mathrm{OE}(-0.621), \mathrm{CE}(0.289)$, coefficient of shoulder asymmetry relative to $\mathrm{C}_{7} \mathrm{OE}(0.114)$, $\mathrm{CE}(0.142)$, primary curvature angle $\mathrm{OE}(-0.266), \mathrm{CE}(0.150)$, length of secondary curvature/total spine length $\mathrm{OE}$ (0.846), CE (0.241), depth of secondary curvature/total spine length OE (0.988), CE (-0.237) (Tab. 4). In the canonical correlation regarding postural stability variables, the largest share regarded: average COP X OE (-0.088), CE (0.054), absolute value of average COP X - OE (-0.793), CE (0.251), forward-backward standard deviation Y 
- OE (-0.590), medium-lateral standard deviation X OE (0.333), average forward-backward speed Y - OE (0.038), CE (-0.364), absolute value of average COP Y (CE) (0.577), average medium-lateral speed X CE (0.028), perimeter ratio (OE) (-0.684), CE (0.971), area ratio (OE) (0.569), CE (-0.524), (Table 4). The high value of the canonical correlation coefficient, despite lack of significance $(\mathrm{R}=0.95521)(p=0.22960)(\mathrm{OE})$ and $(\mathrm{R}=0.95521)$, ( $p=0.27144$ ) (CE) indicates the possibility of a strong correlation between both sets of variables that would be able to be demonstrated with a larger sample size (Table 4).

Table 1. Factorial analysis of body posture variables

(factorial loads - LC)

\begin{tabular}{|c|c|c|c|c|c|}
\hline Body posture variables & $\begin{array}{c}\text { Factor } \\
1\end{array}$ & $\begin{array}{c}\text { Factor } \\
2\end{array}$ & $\begin{array}{c}\text { Factor } \\
\mathbf{3}\end{array}$ & $\begin{array}{c}\text { Factor } \\
4\end{array}$ & $\begin{array}{c}\text { Factor } \\
5\end{array}$ \\
\hline Trunk inclination angle & -0.170 & -0.944 & 0.061 & -0.019 & 0.101 \\
\hline Abs value of trunk inclination angle & -0.135 & -0.926 & 0.035 & 0.061 & 0.111 \\
\hline Alpha angle & -0.168 & -0.173 & 0.919 & 0.001 & -0.043 \\
\hline Beta angle & 0.724 & 0.514 & 0.105 & -0.042 & 0.040 \\
\hline Gamma angle & 0.557 & -0.698 & 0.053 & 0.191 & 0.303 \\
\hline Delta angle & 0.599 & -0.271 & 0.712 & -0.005 & 0.220 \\
\hline Compensation index & 0.526 & -0.393 & -0.604 & 0.154 & 0.261 \\
\hline Absolute value of compensation index & -0.409 & 0.120 & 0.481 & -0.021 & -0.289 \\
\hline Length of chest kyphosis & 0.108 & -0.079 & -0.156 & 0.840 & 0.493 \\
\hline Length of chest kyphosis / Total spine length & -0.046 & -0.002 & 0.053 & 0.049 & 0.809 \\
\hline Angle of chest kyphosis & -0.882 & 0.210 & -0.195 & -0.032 & -0.286 \\
\hline Actual angle of chest kyphosis & 0.384 & -0.159 & -0.125 & 0.458 & 0.730 \\
\hline Actual angle of chest kyphosis / Total spine length & 0.445 & -0.167 & -0.027 & -0.088 & 0.838 \\
\hline Depth of chest kyphosis & 0.874 & 0.296 & -0.086 & 0.036 & 0.270 \\
\hline Depth of chest kyphosis / Total spine length & 0.866 & 0.322 & -0.066 & -0.154 & 0.254 \\
\hline Absolute depth of chest kyphosis / Total spine length & 0.846 & 0.365 & 0.019 & -0.129 & 0.197 \\
\hline Length of lumbar lordosis & -0.075 & 0.005 & 0.045 & 0.960 & -0.144 \\
\hline Length of lumbar lordosis / Total spine length & -0.348 & 0.185 & 0.432 & 0.252 & -0.681 \\
\hline Angle of lumbar lordosis & -0.318 & -0.240 & -0.886 & 0.150 & -0.027 \\
\hline Actual angle of lumbar lordosis & -0.300 & 0.055 & -0.092 & 0.734 & -0.577 \\
\hline Actual angle of lumbar lordosis / Total spine length & -0.434 & 0.141 & 0.059 & 0.128 & -0.847 \\
\hline Depth of lumbar lordosis & 0.324 & 0.675 & 0.504 & 0.229 & -0.043 \\
\hline Depth of lumbar lordosis / Total spine length & 0.263 & 0.696 & 0.562 & 0.013 & -0.133 \\
\hline Baseline value & 5.760 & 4.256 & 3.615 & 2.650 & 4.261 \\
\hline Share & 0.250 & 0.185 & 0.157 & 0.115 & 0.185 \\
\hline Total share of values & 0.250 & 0.435 & 0.593 & 0.708 & 0.893 \\
\hline
\end{tabular}

Table 2. Factorial analysis of scoliotic variables

(factorial loads - LC)

\begin{tabular}{|c|c|c|c|c|c|c|c|}
\hline Scoliotic variables with open eyes & $\begin{array}{c}\text { Fac } \\
\text { tor } \\
1\end{array}$ & $\begin{array}{c}\text { Fac } \\
\text { tor } \\
2\end{array}$ & $\begin{array}{c}\text { Fact } \\
\text { or } \\
3\end{array}$ & $\begin{array}{c}\text { Fact } \\
\text { or } \\
4\end{array}$ & $\begin{array}{c}\text { Factor } \\
5\end{array}$ & $\begin{array}{c}\text { Factor } \\
6\end{array}$ & $\begin{array}{c}\text { Fact } \\
\text { or } \\
7\end{array}$ \\
\hline \multirow[t]{2}{*}{ Shoulder asymmetry - right higher } & 0.2 & 0.2 & 0.16 & 0.02 & -0.035 & -0.106 & 0.83 \\
\hline & 25 & 86 & 9 & 5 & & & 4 \\
\hline \multirow[t]{3}{*}{ Shoulder asymmetry- left higher } & 0.0 & - & 0.09 & - & 0.456 & 0.618 & - \\
\hline & 71 & 0.3 & 6 & 0.06 & & & 0.42 \\
\hline & & 05 & & 4 & & & 9 \\
\hline Shoulder line angle & 0.0 & - & 0.00 & 0.06 & 0.164 & 0.327 & - \\
\hline
\end{tabular}


Absolute shoulder line angle

Shoulder blade asymmetry - right higher

Shoulder blade asymmetry - left higher

Pelvis tilt angle

Absolute pelvis tilt angle

Pelvis rotation angle

Absolute pelvis rotation angle

Coefficient of shoulder asymmetry - KK

Coefficient of shoulder asymmetry relative to $C_{7}$

Length of primary curvature

Length of primary curvature / Total spine length

Depth of primary curvature

Depth of primary curvature / Total spine length

Primary curvature angle

Absolute secondary curvature angle

Length of primary curvature

Length of secondary curvature / Total spine length

Depth of secondary curvature

Depth of secondary curvature / Total spine length

\begin{tabular}{|c|c|c|c|c|c|c|}
\hline 75 & $\begin{array}{l}0.4 \\
06\end{array}$ & 2 & 2 & & & $\begin{array}{c}0.73 \\
3\end{array}$ \\
\hline 0.3 & - & 0.26 & 0.09 & 0.661 & 0.428 & 0.11 \\
\hline \multirow[t]{2}{*}{35} & 0.2 & 3 & 8 & & & 9 \\
\hline & 11 & & & & & \\
\hline 0.0 & 0.0 & 0.20 & 0.07 & 0.020 & -0.778 & - \\
\hline 96 & 34 & 6 & 2 & & & 0.05 \\
\hline & & & & & & \\
\hline 0.1 & 0.1 & 0.05 & 0.25 & 0.015 & 0.786 & - \\
\hline 96 & 39 & 8 & 7 & & & 0.06 \\
\hline 0.0 & 0.1 & - & - & 0.785 & 0.008 & - \\
\hline \multirow[t]{2}{*}{26} & 10 & 0.08 & 0.26 & & & 0.16 \\
\hline & & 0 & 5 & & & 9 \\
\hline - & - & 0.07 & 0.11 & 0.797 & -0.060 & - \\
\hline 0.2 & 0.1 & 6 & 0 & & & 0.10 \\
\hline 66 & 49 & & & & & 8 \\
\hline 0.0 & - & 0.29 & - & -0.111 & -0.266 & - \\
\hline \multirow[t]{2}{*}{15} & 0.2 & 1 & 0.72 & & & 0.17 \\
\hline & 99 & & 6 & & & 7 \\
\hline 0.0 & - & 0.26 & - & -0.148 & 0.389 & 0.68 \\
\hline \multirow[t]{2}{*}{61} & 0.1 & 7 & 0.01 & & & 2 \\
\hline & 71 & & 0 & & & \\
\hline- & - & - & 0.63 & 0.110 & 0.493 & - \\
\hline 0.0 & 0.1 & 0.08 & 4 & & & 0.38 \\
\hline 92 & 64 & 3 & & & & 2 \\
\hline 0.0 & - & - & 0.86 & -0.234 & -0.134 & - \\
\hline \multirow[t]{2}{*}{91} & 0.1 & 0.04 & 7 & & & 0.02 \\
\hline & 97 & 2 & & & & 2 \\
\hline- & 0.0 & 0.13 & - & 0.038 & -0.034 & 0.04 \\
\hline 0.8 & 12 & 3 & 0.09 & & & 1 \\
\hline \multicolumn{2}{|l|}{82} & & 2 & & & \\
\hline- & - & 0.11 & 0.03 & -0.044 & 0.016 & 0.03 \\
\hline 0.9 & 0.0 & 3 & 5 & & & 3 \\
\hline 71 & 60 & & & & & \\
\hline- & 0.0 & 0.96 & - & 0.040 & -0.064 & 0.08 \\
\hline 0.1 & 86 & 0 & 0.10 & & & 5 \\
\hline \multicolumn{2}{|l|}{19} & & 0 & & & \\
\hline- & 0.0 & 0.96 & - & 0.034 & -0.074 & 0.07 \\
\hline 0.1 & 65 & 7 & 0.07 & & & 1 \\
\hline \multicolumn{2}{|l|}{06} & & 2 & & & \\
\hline- & - & - & 0.02 & 0.032 & 0.064 & - \\
\hline 0.0 & 0.9 & 0.09 & 9 & & & 0.13 \\
\hline 36 & 33 & 3 & & & & 6 \\
\hline 0.1 & - & 0.96 & - & 0.059 & 0.006 & 0.12 \\
\hline \multirow[t]{2}{*}{67} & 0.0 & 0 & 0.07 & & & 6 \\
\hline & 07 & & 7 & & & \\
\hline 0.9 & 0.0 & - & - & 0.040 & -0.032 & - \\
\hline \multirow[t]{2}{*}{33} & 68 & 0.11 & 0.08 & & & 0.00 \\
\hline & & 5 & 8 & & & 3 \\
\hline 0.9 & 0.0 & - & - & 0.034 & -0.002 & - \\
\hline \multirow[t]{2}{*}{73} & 46 & 0.12 & 0.03 & & & 0.03 \\
\hline & & 4 & 4 & & & 5 \\
\hline 0.8 & 0.0 & 0.34 & 0.10 & -0.124 & 0.106 & 0.27 \\
\hline 02 & 79 & 1 & 9 & & & 5 \\
\hline 0.8 & 0.0 & 0.32 & 0.12 & -0.129 & 0.112 & 0.24 \\
\hline
\end{tabular}




\begin{tabular}{|c|c|c|c|c|c|c|c|}
\hline & 12 & 65 & 8 & 5 & & & 0 \\
\hline \multirow[t]{3}{*}{ Secondary curvature angle } & 0.2 & 0.9 & 0.08 & - & -0.096 & 0.044 & 0.17 \\
\hline & 66 & 02 & 1 & 0.04 & & & 3 \\
\hline & & & & 9 & & & \\
\hline \multirow[t]{3}{*}{ Absolute secondary curvature angle } & 0.7 & 0.2 & 0.53 & - & -0.046 & 0.064 & 0.13 \\
\hline & 25 & 39 & 9 & 0.06 & & & 7 \\
\hline & & & & 2 & & & \\
\hline \multirow[t]{2}{*}{ Baseline value } & 5.8 & 2.3 & 3.69 & 1.92 & 2.083 & 2.439 & 2.35 \\
\hline & 08 & 96 & 9 & 8 & & & 2 \\
\hline \multirow[t]{2}{*}{ Share } & 0.2 & 0.1 & 0.15 & 0.08 & 0.087 & 0.102 & 0.09 \\
\hline & 42 & 00 & 4 & 0 & & & 8 \\
\hline \multirow[t]{2}{*}{ Total share value } & 0.2 & 0.3 & 0.49 & 0.57 & 0.663 & 0.765 & 0.86 \\
\hline & 42 & 42 & 6 & 6 & & & 3 \\
\hline
\end{tabular}

Table 3. Factorial analysis of postural stability variables with open and closed eyes (OE/CE)

\begin{tabular}{|c|c|c|c|c|c|}
\hline Variables of postural stability with open eyes (OE) & $\begin{array}{c}\text { Fact } \\
\text { or } \\
1\end{array}$ & $\begin{array}{c}\text { Fact } \\
\text { or } \\
2\end{array}$ & $\begin{array}{c}\text { Factor } \\
\mathbf{3}\end{array}$ & $\begin{array}{c}F \\
\text { ac } \\
\text { to } \\
r \\
4\end{array}$ & $\begin{array}{c}\text { Fact } \\
\text { or } \\
5\end{array}$ \\
\hline Average COP X & $\begin{array}{c}- \\
0.20 \\
3\end{array}$ & $\begin{array}{c}- \\
0.12 \\
6\end{array}$ & 0.002 & $\begin{array}{l}0 . \\
3 \\
3 \\
8\end{array}$ & $\begin{array}{c}- \\
0.82 \\
9\end{array}$ \\
\hline Absolute value of average COP X & $\begin{array}{c}- \\
0.02 \\
1\end{array}$ & $\begin{array}{c}0.88 \\
6\end{array}$ & -0.183 & $\begin{array}{l}0 . \\
2 \\
4 \\
7\end{array}$ & $\begin{array}{c}0.20 \\
3\end{array}$ \\
\hline Average COP Y & $\begin{array}{c}- \\
0.16 \\
0\end{array}$ & $\begin{array}{c}0.39 \\
9\end{array}$ & -0.154 & $\begin{array}{l}0 . \\
0 \\
6 \\
3\end{array}$ & $\begin{array}{c}0.82 \\
2\end{array}$ \\
\hline Absolute value of average COP Y & $\begin{array}{c}- \\
0.08 \\
3\end{array}$ & $\begin{array}{c}0.88 \\
2\end{array}$ & 0.074 & $\begin{array}{l}- \\
0 . \\
2 \\
8 \\
0\end{array}$ & $\begin{array}{c}0.22 \\
5\end{array}$ \\
\hline Forward-backward standard deviation Y & $\begin{array}{c}0.19 \\
7\end{array}$ & $\begin{array}{c}- \\
0.10 \\
6\end{array}$ & 0.883 & $\begin{array}{l}0 . \\
3 \\
4 \\
1\end{array}$ & $\begin{array}{c}- \\
0.11 \\
3\end{array}$ \\
\hline Medium-lateral standard deviation X & $\begin{array}{c}0.96 \\
1\end{array}$ & $\begin{array}{c}0.02 \\
9\end{array}$ & 0.130 & $\begin{array}{l}0 . \\
0 \\
3 \\
1\end{array}$ & $\begin{array}{c}0.03 \\
1\end{array}$ \\
\hline Average forward-backward speed Y & $\begin{array}{c}0.27 \\
4\end{array}$ & $\begin{array}{c}0.03 \\
0\end{array}$ & 0.379 & $\begin{array}{l}0 . \\
8 \\
1 \\
7\end{array}$ & $\begin{array}{c}- \\
0.20 \\
0\end{array}$ \\
\hline Average medium-lateral speed X & $\begin{array}{c}0.89 \\
4\end{array}$ & $\begin{array}{c}- \\
0.18 \\
8\end{array}$ & -0.049 & $\begin{array}{l}0 . \\
3 \\
0 \\
4\end{array}$ & $\begin{array}{c}0.01 \\
3\end{array}$ \\
\hline Perimeter ratio & 0.57 & - & 0.269 & 0 . & - \\
\hline
\end{tabular}




\begin{tabular}{|c|c|c|c|c|c|}
\hline & 8 & 0.03 & & 7 & 0.14 \\
\hline & & 9 & & 3 & 2 \\
\hline & & & & 6 & \\
\hline Ellipse area & 0.80 & - & 0.462 & 0. & 0.02 \\
\hline & 9 & 0.01 & & 2 & 8 \\
\hline & & 0 & & 8 & \\
\hline & & & & 2 & \\
\hline Baseline value & 2.89 & 1.78 & 1.292 & 1. & 1.53 \\
\hline & 9 & 7 & & 7 & 0 \\
\hline & & & & 5 & \\
\hline & & & & 7 & \\
\hline Share & 0.29 & 0.17 & 0.129 & 0. & 0.15 \\
\hline & 0 & 9 & & 1 & 3 \\
\hline & & & & 7 & \\
\hline & & & & 6 & \\
\hline Totalled share of values & 0.29 & 0.46 & 0.598 & 0. & 0.92 \\
\hline & 0 & 9 & & 7 & 6 \\
\hline & & & & 7 & \\
\hline & & & & 3 & \\
\hline Variables of postural stability with open eyes (CE) & Fact & Fact & Factor & $\mathrm{F}$ & Fact \\
\hline & or & or & 3 & ac & or \\
\hline & 1 & 2 & & to & 5 \\
\hline & & & & $\mathrm{r}$ & \\
\hline & & & & 4 & \\
\hline Average COP X & 0.09 & 0.08 & 0.123 & $\mathbf{0 .}$ & 0.04 \\
\hline & 5 & 3 & & 9 & 4 \\
\hline & & & & 3 & \\
\hline & & & & 7 & \\
\hline Absolute value of average COP X & - & - & -0.902 & - & 0.01 \\
\hline & 0.05 & 0.10 & & 0 . & 5 \\
\hline & 3 & 9 & & 1 & \\
\hline & & & & 1 & \\
\hline & & & & 5 & \\
\hline Average COP Y & - & 0.82 & 0.306 & - & 0.30 \\
\hline & 0.14 & 1 & & 0 . & 8 \\
\hline & 6 & & & 0 & \\
\hline & & & & 2 & \\
\hline & & & & 6 & \\
\hline Absolute value of average COP Y & 0.10 & 0.92 & -0.090 & 0 . & - \\
\hline & 0 & 7 & & 1 & 0.14 \\
\hline & & & & 4 & 6 \\
\hline & & & & 4 & \\
\hline Forward-backward standard deviation $\mathrm{Y}$ & 0.89 & 0.03 & -0.170 & 0 . & 0.22 \\
\hline & 1 & 4 & & 1 & 1 \\
\hline & & & & 8 & \\
\hline & & & & 2 & \\
\hline Medium-lateral standard deviation X & 0.49 & 0.16 & -0.393 & 0 . & $\mathbf{0 . 5 1}$ \\
\hline & 6 & 4 & & 3 & 5 \\
\hline & & & & 8 & \\
\hline & & & & 0 & \\
\hline Average forward-backward speed $\mathrm{Y}$ & 0.97 & - & 0.111 & 0 . & 0.05 \\
\hline & 4 & 0.04 & & 0 & 3 \\
\hline & & 7 & & 2 & \\
\hline & & & & 2 & \\
\hline Average medium-lateral speed $\mathrm{X}$ & 0.52 & 0.00 & 0.059 & - & 0.77 \\
\hline & 1 & 3 & & 0 . & 3 \\
\hline
\end{tabular}




\begin{tabular}{|c|c|c|c|c|c|}
\hline & & & & $\begin{array}{l}0 \\
0 \\
7\end{array}$ & \\
\hline \multirow[t]{4}{*}{ Perimeter ratio } & 0.90 & - & 0.133 & 0 . & 0.34 \\
\hline & 5 & 0.05 & & 0 & 2 \\
\hline & & 1 & & 0 & \\
\hline & & & & 6 & \\
\hline \multirow[t]{4}{*}{ Ellipse area } & 0.67 & 0.14 & -0.276 & 0 . & 0.48 \\
\hline & 1 & 2 & & 3 & 5 \\
\hline & & & & 4 & \\
\hline & & & & 0 & \\
\hline \multirow[t]{4}{*}{ Baseline value } & 3.57 & 1.60 & 1.224 & 1. & 1.38 \\
\hline & 3 & 5 & & 2 & 5 \\
\hline & & & & 0 & \\
\hline & & & & 6 & \\
\hline \multirow[t]{4}{*}{ Share } & 0.35 & 0.16 & 0.122 & 0 . & 0.13 \\
\hline & 7 & 0 & & 1 & 9 \\
\hline & & & & 2 & \\
\hline & & & & 1 & \\
\hline \multirow[t]{4}{*}{ Totalled share of values } & 0.35 & 0.51 & 0.640 & 0. & 0.89 \\
\hline & 7 & 8 & & 7 & 9 \\
\hline & & & & 6 & \\
\hline & & & & 1 & \\
\hline
\end{tabular}

Table 4. Summary of canonical analysis

\begin{tabular}{|c|c|c|c|c|}
\hline & Left set & & Right set & \\
\hline $\begin{array}{l}\text { Number of } \\
\text { variables }\end{array}$ & 12 & \multirow[t]{4}{*}{$\begin{array}{l}\text { Canonical } \\
\text { weights }\end{array}$} & 7 & \multirow[t]{4}{*}{$\begin{array}{l}\text { Canonical } \\
\text { weights }\end{array}$} \\
\hline $\begin{array}{c}\text { Isolated } \\
\text { variation }\end{array}$ & $66.04 \%$ & & $100.00 \%$ & \\
\hline $\begin{array}{c}\text { Total } \\
\text { redundancy }\end{array}$ & $31.54 \%$ & & $46.73 \%$ & \\
\hline Variables & $\begin{array}{l}\text { Body posture and scoliotic } \\
\text { variables }(\mathrm{OE})\end{array}$ & & $\begin{array}{l}\text { Variables of postural stability } \\
\text { with open eyes }\end{array}$ & \\
\hline 1 & Trunk inclination angle & 0.555 & Average COP X & -0.088 \\
\hline 2 & Alpha angle & -0.070 & $\begin{array}{l}\text { Absolute value of Average } \\
\text { COP X }\end{array}$ & -0.793 \\
\hline 3 & Chest kyphosis angle & 0.216 & $\begin{array}{l}\text { Forward-Backward Standard } \\
\text { Deviation Y }\end{array}$ & -0.590 \\
\hline 4 & Length of lumbar lordosis & 0.409 & $\begin{array}{ll}\text { Medium-Lateral } & \text { Standard } \\
\text { Deviation X } & \\
\end{array}$ & 0.333 \\
\hline 5 & $\begin{array}{l}\text { Length of lumbar lordosis / Total } \\
\text { spine length }\end{array}$ & -0.339 & $\begin{array}{l}\text { Average Forward-Backward } \\
\text { Speed Y }\end{array}$ & 0.038 \\
\hline 6 & $\begin{array}{l}\text { Shoulder asymmetry - right } \\
\text { higher }\end{array}$ & -0.199 & Perimeter ratio & -0.684 \\
\hline 7 & $\begin{array}{l}\text { Shoulder blade asymmetry - } \\
\text { left higher }\end{array}$ & -0.641 & Area ratio & 0.569 \\
\hline 8 & Absolute of pelvis tilt angle & -0.621 & & \\
\hline 9 & $\begin{array}{l}\text { Coefficient of shoulder } \\
\text { asymmetry relative to } \mathrm{C}_{7}\end{array}$ & 0.114 & & \\
\hline 10 & Primary curvature angle & -0.266 & & \\
\hline 11 & $\begin{array}{l}\text { Length of secondary curvature } \\
\text { /Total spine length }\end{array}$ & -0.846 & & \\
\hline 12 & Depth of secondary curvature & 0.988 & & \\
\hline
\end{tabular}


/Total spine length

$$
\boldsymbol{R}=0.92214 ; \boldsymbol{C h i}^{2}(84)=93.268 ; \boldsymbol{p}=0.22960
$$

\begin{tabular}{|c|c|c|c|c|}
\hline $\begin{array}{l}\text { Number of } \\
\text { variables }\end{array}$ & 12 & $\begin{array}{l}\text { Canonical } \\
\text { weights }\end{array}$ & 7 & $\begin{array}{l}\text { Canonical } \\
\text { weights }\end{array}$ \\
\hline $\begin{array}{c}\text { Isolated } \\
\text { variation } \\
\end{array}$ & $61.67 \%$ & & $100.00 \%$ & \\
\hline $\begin{array}{c}\text { Total } \\
\text { redundancy }\end{array}$ & $28.80 \%$ & & $39.27 \%$ & \\
\hline Variables & $\begin{array}{c}\text { Body posture and scoliotic } \\
\text { variables } \\
\text { (CE) }\end{array}$ & & $\begin{array}{l}\text { Variables of postural stability } \\
\text { with closed eyes }\end{array}$ & \\
\hline 1 & Trunk inclination angle & 0.243 & Average COP X & 0.054 \\
\hline 2 & Alpha angle & -0.422 & $\begin{array}{l}\text { Absolute value of Average } \\
\text { COP X }\end{array}$ & 0.251 \\
\hline 3 & Chest kyphosis angle & -0.347 & $\begin{array}{l}\text { Absolute value of Average } \\
\text { COPY }\end{array}$ & 0.577 \\
\hline 4 & Length of lumbar lordosis & 0.107 & $\begin{array}{l}\text { Average Forward-Backward } \\
\text { Speed Y }\end{array}$ & -0.364 \\
\hline 5 & $\begin{array}{l}\text { Length of lumbar lordosis / } \\
\text { Total spine length }\end{array}$ & 0.864 & $\begin{array}{ll}\text { Average } & \text { Medium-Lateral } \\
\text { Speed X } & \\
\end{array}$ & 0.028 \\
\hline 6 & $\begin{array}{l}\text { Shoulder asymmetry - right } \\
\text { higher }\end{array}$ & -0.271 & Perimeter Ratio & 0.971 \\
\hline 7 & $\begin{array}{l}\text { Shoulder blade asymmetry - } \\
\text { left higher }\end{array}$ & 0.392 & Area Ratio & -0.524 \\
\hline 8 & Absolute of pelvis tilt angle & 0.289 & & \\
\hline 9 & $\begin{array}{l}\text { Coefficient of shoulder } \\
\text { asymmetry relative to } \mathrm{C}_{7}\end{array}$ & 0.142 & & \\
\hline 10 & Primary curvature angle & 0.150 & & \\
\hline 11 & $\begin{array}{l}\text { Length of secondary curvature / } \\
\text { Total spine length }\end{array}$ & 0.241 & & \\
\hline 12 & $\begin{array}{l}\text { Depth of secondary curvature } \\
\text { /Total spine length }\end{array}$ & -0.237 & & \\
\hline
\end{tabular}

\section{Discussion}

Many studies have shown that people with scoliotic changes are characterized by worse postural stability compared to healthy individuals $[18,19]$. In other studies evaluating body posture and balancing responses, there were no differences between individuals with idiopathic scoliosis and the control group in the assessment of static balance. On the other hand, considerable divergences in the assessment of dynamic balance have been observed, indicating that dynamic proprioception is related to body posture [20]. Other researchers assessed postural control with open and closed eyes on the Kistler Power Platform in idiopathic scoliosis and among healthy individuals. They demonstrated a weaker ability to maintain balance in people with scoliosis compared to healthy individuals. According to other authors, a complex system that regulates balancing and postural reactions as well as arbitrary movements in idiopathic scoliosis may be disturbed [21]. Other studies have examined the impact of weight training on improving static and dynamic balance in children with developmental coordination disorders. The study involved children aged 7 to 9 . It has been shown that strength training significantly increased muscle strength in children with coordination disorders and improved their stability but did not significantly affect their dynamic balance [22]. In turn, other researchers assessed the relationship between body posture and static balance among children with sensory-motor deficits. Balance was evaluated with the BTS P-6000 platform on a stable surface, with open and closed eyes. The Nikon D-5000 digital camera was used to evaluate body posture, which displayed images of body posture in the frontal and sagittal planes. Results showed that children with cerebral palsy had the 
highest number of abnormalities both in the sagittal and frontal planes compared to the other groups. In case of analysis of equivalent reactions in the sagittal plane, the greatest instability in the open and closed eyes test was observed in children with mild mental retardation, and with closed eyes, in the case of visual impairment. In the frontal plane, the greatest problems maintaining balance occurred in individuals with cerebral palsy and mild mental retardation. The best results were obtained by persons with hearing impairments [23]. The main clinical problem of children with scoliotic changes is postural hypothyroidism, disturbing the development of central stabilization in the body [24]. Re-education of the postural system, which is the primary goal of scoliosis therapy, is basically developing the possibility of body stabilization [25]. The correct central stabilization in each plane is the so-called postural equalization [26]. Postural alignment is the correct alignment of individual parts of the body in static and moving positions occurring in all planes [27]. Postural alignment in the frontal plane is the symmetry of the individual right and left segments of the body, which is accompanied by the axial alignment of the knees [28]. In the transverse plane, the expression of postural alignment is balance in rotational movement tension of the trunk and the intermediate positioning of the lower limbs in the hip joints between external and internal rotation [29]. Scoliosis is just a symptom, an external manifestation of unrecognized pathology [30]. More and more followers have a multifactorial concept including genetically conditioned CNS changes that cause dysfunctions in the postural system [31]. From a neuronal developmental point of view, primary scoliosis is a central disorder of postural tension, and only afterwards are there consequences of biomechanical compensation [32]. In our opinion, scoliosis is a disorder associated with dysfunction of the body caused by improper CNS functioning [33]. This dysfunction reduces the tension of postural muscles and disrupts the stabilization of the body. These changes are primary and preceded by external symptoms of scoliosis.

\section{Conclusions}

In the canonical analysis of body posture variables, the highest share comprised of: trunk inclination angle, alpha angle, chest kyphosis angle, length of lumbar lordosis, length of lumbar lordosis/total spine length, shoulder asymmetry - right higher, shoulder asymmetry - left higher, absolute of pelvis tilt angle, coefficient of shoulder asymmetry relative to $\mathrm{C}_{7}$, primary curvature angle, length of secondary curvature/total spine length, depth of secondary curvature/total spine length. In the canonical analysis of postural stability variables, the largest share consisted of: average COP X (OE, CE), absolute value of average COP X $(\mathrm{OE}, \mathrm{CE})$, forward-backward standard deviation $\mathrm{Y}(\mathrm{OE})$, medium-lateral standard deviation $\mathrm{X}(\mathrm{OE})$, average forward-backward speed $\mathrm{Y}(\mathrm{OE}, \mathrm{CE})$, absolute value of average $\mathrm{COPY}(\mathrm{CE})$, average medium-lateral speed $\mathrm{X}(\mathrm{CE})$, perimeter ratio $(\mathrm{OE}, \mathrm{CE})$, area ratio (OE, CE). High values of canonical correlation coefficients, despite lack of significance, indicate the possibility of strong a correlation between body postural variables and postural stability that can be demonstrated with a greater sample size.

\section{Formatting of Funding Sources}

This research did not receive any specific grant from funding agencies in the public, commercial, or not-for-profit sectors.

\section{References}

Alves, VLDS, \& Avanzi, O. (2016). Respiratory muscle strength in idiopathic scoliosis after training program. Acta Ortop Bras, 24(6), 296-299.

Bergmark, A. (1989). Stability of the lumbar spine. A study in mechanical engineering. Acta Orthop. Scand. Suppl, 230, 1-54.

Borges, P. A., Zelada, F. G. B., Dos, Santos Barros TF, \& Letaif, O. B, et al. (2017). A Comparative Study of Sagittal Balance in Patients with Neuromuscular Scoliosis. Clinics, 72(8), 481-484.

Brink, R. C., Wijdicks, S. P. J., Tromp, I. N., Schlösser, T. P. C., \& Kruyt, M. C., et al. (2017). A reliability and validity study for different coronal angles using ultrasound imaging in adolescent idiopathic scoliosis. Spine J; 19. pii: S1529-9430(17)31063-X. https:// doi: 10.1016/j.spinee.2017.10.012.

Bruggi, M., Lisi, C., Rodigari, A., Nava, M., Carlisi, E., \& Dalla, T. E. (2014). Monitoring iliopsoas muscle contraction in idiopathic lumbar scoliosis patients. G Ital Med Lav Ergon, 36(3), 186-191.

Burwell, R. G., Dangerfield, P. H., Moulton, A., \& Anderson, S. I. (2008). Etiologic theories of idiopathic scoliosis: autonomic nervous system and the leptin-sympathetic nervous system concept for the pathogenesis of adolescent idiopathic scoliosis. Stud Health Technol Inform, 140, 197-207.

Chang, Y. T., Meng, L. F., Chang, C. J., Lai, P. L., Lung, C. W., \& Chern, J. S. (2017). Effect of Postural Control Demands on Early Visual Evoked Potentials during a Subjective Visual Vertical Perception Task in 
Adolescents with Idiopathic Scoliosis. Front Hum Neurosci, 30(11), 326.

Giampietro, P. F. (2015). SNPping away at the genetic basis of adolescent idiopathic scoliosis. Ann Transl Med., 3(1), S26. https://doi:10.3978/j.issn.2305-5839.2015.02.34.

Girardo, M., Bettini, N., Dema, E., \& Cervellati, S. (2011). The role of melatonin in the pathogenesi/s of adolescent idiopathic scoliosis (AIS). Eur Spine J., 20(1), S68-74. https:/doi:10.1007/ s00586-011-1750-5.

Grivas, T. B., Burwell, R. G., Kechagias, V., Mazioti, C., Fountas, A., Kolovou, D., \& Christodoulou, E. (2016). Idiopathic and normal lateral lumbar curves: muscle effects interpreted by 12th rib length asymmetry with pathomechanic implications for lumbar idiopathic scoliosis. Scoliosis Spinal Disord, 11(2), 35.

Gur, G., Dilek, B., Ayhan, C., Simsek, E., Aras, O., Aksoy, S., \& Yakut, Y. (2015). Effect of a spinal brace on postural control in different sensory conditions in adolescent idiopathic scoliosis: a preliminary analysis. Gait Posture, 41(1), 93-99.

Haumont, T., Gauchard, G. C., Lascombes, P., \& Perrin, P. P. (2011). Postural instability in early - stage idiopathic scoliosis in adolescent girls. Spine, 36(13), 847-854.

Karimi, M. T., Kavyani, M., \& Kamali, M. (2016). Balance and gait performance of scoliotic subjects: A review of the literature. J Back Musculoskelet Rehabil, 29(3), 403-415.

Kepler, C. K., Meredith, D. S., Green, D. W., \& Widmann, R. F. (2012). Long-term outcomes after posterior spine fusion for adolescent idiopathic scoliosis. Curr Opin Pediatr, 24(1), 68-75.

Klavina, A., \& Galeja, Z. (2017). Relationship between postural alignment in standing by photogrammetry and static balance in children with disabilities. Society. Integration. Education. Proccedings of the International Scientific Conference, 3, 359-367.

Kordi, H., Sohrabi, M., Saberi Kakhki, A., \& Attarzadeh, H. S. R. (2016). The effect of strength training based on proces approach intervention on balance of children with developmental coordination disorder. Arch Argent Pediatr; 1, 114(6), 526-533.

Kotwicki, T., Chowanska, J., Kinel, E., Czaprowski, D., Tomaszewski, M., \& Janusz, P. (2013). Optimal management of idiopathic scoliosis in adolescence. Adolesc Health Med Ther, 4, 59-73.

Kubota, K., Doi, T., Murata, M., Kobayakawa, K., \& Matsumoto, Y. et al. (2013). Disturbance of rib cage development causes progressive thoracic scoliosis: the creation of a nonsurgical structural scoliosis model in mice. J Bone Joint Surg Am; 18, 95(18), e130. https://doi: 10.2106/JBJS.L.01381.

Le Berre, M., Guyot, M. A., Agnani, O., Bourdeauducq, I., \& Versyp, M. C., et al. (2017). Clinical balance tests, proprioceptive system and adolescent idiopathic scoliosis. Eur Spine J., 26(6), 1638-1644.

Mayes, T., Anadio, J. M., \& Sturm, P. F. (2017). Prevalence of Vitamin D Deficiency in Pediatric Patients With Scoliosis Preparing for Spinal Surgery. Spine Deform, 5(6). 369-373.

Monticone, M., Ambrosini, E., Cazzaniga, D., Rocca, B., Motta, L., \& Cerri, C., et al. (2016). Adults with idiopathic scoliosis improve disability after motor and cognitive rehabilitation: results of a randomised controlled trial. Eur Spine J., 25(10), 3120-3129.

Nault, M. L., Allard, P., Hinse, S., Le Blanc, R., \& Caron, O., et al. (2002). Relations between standing stability and body posture parameters in adolescent idiopathic scoliosis. Spine; 1, 27(17), 1911-1917.

Park, J. H., Jeon, H. S., \& Park, H. W. (2017). Effects of the Schroth exercise on idiopathic scoliosis: a metaanalysis. Eur J Phys Rehabil Med.; 2. https:// doi: 10.23736/S1973-9087.17.04461-6.

Pialasse, J. P., Mercier, P., Descarreaux, M., \& Simoneau, M. (2016). Sensorimotor Control Impairment in Young Adults With Idiopathic Scoliosis Compared With Healthy Controls. J Manipulative Physiol Ther, 39(7), 473 479.

Porte, M., Patte, K., Dupeyron, A., \& Cottalorda, J. (2016). Exercise therapy in the treatment of idiopathic adolescent scoliosis: Is it useful ? Arch Pediatr, 23(6): 624-8.

Putzier, M., Groß, C., Zahn, R. K., Pumberger, M., \& Strube, P. (2016). Characteristics of neuromuscular scoliosis. Orthopade, 45(6), 500-508.

Ramirez, J. M., \& Eberson, C. P. (2017). The Role of Rehabilitation in the Management of Adolescent Idiopathic Scoliosis. R I Med J., 100(11), 22-25.

Scaramuzzo, L., Giudici, F., Bongetta, D., Caboni, E., Minoia, L., \& Zagra, A. (2017). Thoraco-lumbar selective fusion in adolescent idiopathic scoliosis with Lenke $\mathrm{C}$ modifier curves: clinical and radiographic analysis at 
10-year follow-up. Eur Spine J., 25. https://doi:10.1007/s0 0586-017-5152-1.

Szopa, A., \& Domagalska-Szopa, M. (2015). Postural stability in children with hemiplegia estimated for three postural conditions: standing, sitting and kneeling. Res Dev Disabil, 39, 67-75.

Weiss, H. R. (2016). Current knowledge on physiotherapy for scoliosis. Orthopade, 45(6), 549-550.

Wilczyński, J. (2014). Body posturę defects and mean sway X and mean sway Y in girls and boys of school age. Journal of Polish Society of Child Neurologist, 23(46), 27-33.

Wong, C., Gosvig, K., \& Sonne-Holm, S. (2017). The role of the paravertebral muscles in adolescent idiopathic scoliosis evaluated by temporary paralysis. Scoliosis Spinal Disord, 10, 12-33. https://doi:10.1186/s13013017-0138-7.

Yen, T. C., Toosizadeh, N., Howe, C., Dohm, M., Mohler, J., \& Najafi, B. (2016). Postural Balance Parameters as Objective Surgical Assessments in Low Back Disorders: A Systematic Review. J Appl Biomech, 32(3), 316323.

\section{Copyrights}

Copyright for this article is retained by the author(s), with first publication rights granted to the journal.

This is an open-access article distributed under the terms and conditions of the Creative Commons Attribution license (http://creativecommons.org/licenses/by/4.0/). 